\title{
IL-13 alters mucociliary differentiation and ciliary beating of human respiratory epithelial cells
}

\author{
Jamila Laoukili, ${ }^{1}$ Eric Perret, ${ }^{2}$ Tom Willems, ${ }^{3}$ Adrian Minty, ${ }^{2}$ Eef Parthoens, ${ }^{3}$ \\ Odile Houcine, ${ }^{1}$ Andre Coste,${ }^{4}$ Mark Jorissen, ${ }^{3}$ Francelyne Marano, ${ }^{1}$ Daniel Caput, ${ }^{2}$ \\ and Frédéric Tournier ${ }^{1}$
}

${ }^{1}$ Laboratoire de Cytophysiologie et Toxicologie Cellulaire, Université Paris 7, Paris, France
${ }^{2}$ Sanofi-Synthelabo Recherche, Labège Innopole, France
32aboratory for Experimental Otorhinolaryngologica, Universitaire Ziekenhuizen Leuven, Leuven, Belgium
${ }^{4}$ INSERM U296, Faculté de Médecine Paris 12, Créteil, France

Address correspondence to: Frédéric Tournier, Laboratoire de Cytophysiologie et Toxicologie Cellulaire, Université Paris 7, Tour 53-54, 3ème étage, case 7073, 2 place Jussieu, 75251 Paris cedex 05, France.

Phone: 33-1-44-27-60-72; Fax: 33-1-44-27-69-99; E-mail: f-tournier@paris7.jussieu.fr.

Received for publication June 19, 2001, and accepted in revised form October 9, 2001.

\begin{abstract}
In animal models of asthma, interleukin-13 (IL-13) induces goblet cell metaplasia, eosinophil infiltration of the bronchial mucosa, and bronchial hyperreactivity, but the basis of its effects on airway epithelia remain unknown. Lesions of the epithelial barrier, frequently observed in asthma and other chronic lung inflammatory diseases, are repaired through proliferation, migration, and differentiation of epithelial cells. An inflammatory process may then, therefore, influence epithelial regeneration. We have thus investigated the effect of IL-13 on mucociliary differentiation of human nasal epithelial cells in primary culture. We show that IL-13 alters ciliated cell differentiation and increases the proportion of secretory cells. IL-13 downregulates the actin-binding protein ezrin and other cytoskeletal components. IL-13 also impairs lateral cell contacts and interferes with the apical localization of ezrin seen in differentiated ciliated cells. In addition, an IL-4 antagonistic mutant protein (Y124D), which binds to the IL-4 receptor $\alpha$ subunit, a common chain of IL-4 and IL-13 receptors, inhibits IL-13's effects. IL-13 also decreases ciliary beat frequency in a time- and dose-dependent manner. These results suggest that, in human allergic asthmatic responses, IL-13 affects both ciliated and secretory cell differentiation, leading to airway damage and obstruction.
\end{abstract}

J. Clin. Invest. 108:1817-1824 (2001). DOI:10.1172/JCI200113557.

\section{Introduction}

The lining airway tissue consists of a pseudostratified columnar epithelium that contains three main cell types: basal, secretory, and ciliated cells. The upper respiratory epithelium forms a continuous layer, thereby protecting the underlying tissue from various noxious agents. A continuous regeneration process that involves proliferation, migration, and differentiation of adjacent epithelial cells, as well as several cell-cell adhesion mechanisms, maintain the integrity of this epithelium. The mucosecretory phenotype is largely controlled by environmental cues. The airway epithelium responds to infectious agents, particles, pollutants, and allergens by increasing production of mucus, cytokines, reactive oxygen, and nitrogen species (see ref. 1 for a review). Moreover, environmental signals can significantly alter epithelium functions and lead to long-term airway hyperresponsiveness (AHR). Even though mechanisms that endorse longterm pulmonary diseases are not understood, in animal models IL-13 has recently emerged as a pivotal molecule to promote AHR (2). This cytokine was shown to be responsible for increased mucus production, goblet cell hyperplasia, and fibrosis.
Many questions are unanswered regarding source and regulation of IL-13 production as well as its potential functions on epithelial cells. IL-13 is generally described as a Th2-type cytokine that can be coexpressed with IL-4 and IL-5 (3). Recent studies have also demonstrated that alveolar macrophages of patients with lung fibrosis (4) or asthma (5) express a high level of IL-13. A shared IL-4/IL-13 receptor (IL-4/IL-13 R) that is composed of both IL-13R alpha 1 (IL-13R $\alpha 1$ ) and IL-4R alpha (IL-4R $\alpha$ ) chains mediates IL-13 activities by STAT-6 activation (5-9). In IL-4R $\alpha$-deficient or STAT-6-deficient mice, in which both IL-4 and IL-13 signaling are impaired, allergen-induced goblet cell metaplasia, eosinophilic inflammation, and IgE production are largely inhibited $(10,11)$. Recent data suggest that airway epithelial cells are direct targets for IL-13 $(12,13)$. But the use of epithelial cell lines in these studies could not really address the question of how IL-13 could contribute to AHR. Does IL-13 only influence the activities of fully differentiated epithelial cells such as mucus secretion, or does the cytokine also interfere with the differentiation process of epithelial stem cells?

To explore the mechanisms by which IL-13 could alter airway epithelial cell differentiation, we examined its 
effect on mucociliary differentiation (MCD) of human nasal epithelial (HNE) cells. We have developed a primary culture where "basal-like" epithelial cells proliferate on a collagen gel matrix and further differentiate as epithelial cell spheroids, a highly efficient method to obtain and quantify MCD $(14,15)$. Here, we show that IL-13 dramatically alters the ciliated cell differentiation process and largely increases the proportion of secretory cells. These effects are mediated by a common receptor chain for IL-13 and IL-4. IL-13 affects the expression of ezrin, an actin-binding associated protein, and interferes with its apical localization during ciliated cell differentiation. At the ultrastructural level, IL-13 interferes with epithelial cell polarization, altering the apical and basolateral membrane-specification domains of epithelial cells. Moreover, IL-13 decreases ciliary beat frequency (CBF) of ciliated epithelial cells in a time- and dose-dependent way.

\section{Methods}

Cytokines and $A b$ 's. IL-4 and IFN- $\gamma$ were purchased from R\&D Systems Europe Ltd. (Abingdon, United Kingdom). The IL-4 mutant protein (Y124D) was a kind gift from W. Sebald (Würzburg University, Würzburg, Germany). Human recombinant IL-13 was purified from Escherichia coli (5) or, for some experiments, was purchased from Sigma (St. Quentin Fallavier, France). Anti-glutamylated tubulin mAb (GT335) was a gift from P. Denoulet (Université Paris 6, Paris, France). A $\mathrm{mAb}$ raised against human gastric mucins (anti-M1 $\mathrm{mAb}$ ) was a gift from J. Bara (Hospital Saint-Antoine, Paris, France). Polyclonal anti-ezrin and anti-ZO-1 Ab's were a gift from M. Arpin (Institut Curie, Paris, France).

Cell culture. Primary cell culture was achieved as initially described in Jorissen et al. (16) with slight modifications. Nasal tissue (mainly polyps or turbinates) was surgically removed, and epithelial cells were dissociated from the tissue by using $0.1 \%$ filtered pronase (protease XIV: Sigma) overnight at $4^{\circ} \mathrm{C}$ in Ham's F12-DMEM 1/1 (Gibco; Invitrogen Sarl, Cergy-Pontoise, France) supplemented with antibiotics $(50 \mathrm{U} / \mathrm{ml}$ penicillin and 50 $\mu \mathrm{g} / \mathrm{ml}$ streptomycin), $1 \%$ Fungizone, and $2 \%$ Ultroser G (UG; Invitrogen Sarl). The cell suspension $(10 \mathrm{ml})$ obtained after centrifugation, and resuspension of the pellet was pipetted into T-25 uncoated culture flasks. In this step (preplating), the fibroblasts were separated from the epithelial cells because of their higher attachment ratio $(90 \% / 5 \%)$. The cell suspension obtained after preplating was filtered and dispersed on $0.2 \%$, thick, type I collagen gels obtained from rat tails in T-25 or T-75 culture flasks $\left(10^{4} \mathrm{cells} / \mathrm{cm}^{2}\right)$. The monolayer medium $(2 \%$ UG in DMEM-F12) was replaced three times a week.

Suspension culture. After 2-3 weeks the confluent monolayers consisted of "basal-like" epithelial cells. Collagenase (330 IU/ml; Sigma) was added to resolve the collagen gel and to release the epithelial cells as cell sheets in suspension. Cells were washed three times with monolayer medium to make sure that the collagenase was removed. The suspension of cell sheets was pipetted into T-25 uncoated culture flasks. The cells were placed on a continuous rotating shaker $(80 \mathrm{rpm}$; Fisher Bioblock Scientific, Rungis, France) at $37^{\circ} \mathrm{C}$ for 1 week. Cell sheets formed stable aggregates (epithelial spheroids), and ciliogenesis began. The culture medium (10 $\mathrm{ml}$ ) was replaced each day, the first 2 days with monolayer medium and afterward with suspension medium. In suspension medium, $2 \%$ UG was replaced by $10 \%$ NuSerum (NuS; Becton Dickinson Co., Franklin Lakes, New Jersey, USA). After the first week, the T-25 flasks were then put in an incubator $\left(37^{\circ} \mathrm{C}, 98 \%\right.$ humidity, and $5 \% \mathrm{CO}_{2}$ ). During the second and third weeks, the culture medium was replaced three times a week with suspension medium $(5-10 \mathrm{ml})$. After 3 weeks, ciliogenesis resulted in $20-60 \%$ ciliated cells (14).

Immunofluorescence. Epithelial spheroids were washed in PBS, fixed in 3.7\% paraformaldehyde in PBS for 20 minutes, and postfixed in methanol $\left(-20^{\circ} \mathrm{C}\right)$. Immunofluorescence (IF) was performed as described in Laoukili et al. (15). Primary Ab's were used at the following dilutions: GT335 (1:10,000), anti-M1 mAb (undiluted supernatant), anti-ezrin $A b(1: 5,000)$, and anti-ZO1 Ab (1:100). The secondary Ab's were FITCconjugated anti-rabbit and rhodamine-conjugated anti-mouse Ab's (Sigma; 1:200).

Flow cytometry. Cells were dissociated from epithelial spheroids with $0.2 \%$ trypsin in a cell dissociation buffer (Sigma) and fixed in $-20^{\circ} \mathrm{C}$ methanol. Immunostaining of epithelial cells using GT335 mAb or anti-M1 $\mathrm{mAb}$ and flow cytometric analyses were performed as described previously (14).

Protein analysis. Total protein extracts were prepared from HNE cells in SDS-PAGE sample buffer and resolved by electrophoresis in a $8 \%$ or $10 \%$ SDS-PAGE. After transfer onto a nitrocellulose filter, immunodetection was performed as described previously (14) using GT335 mAb (1:10,000), anti- $\alpha$-tubulin mAb $(1: 1,000)$, anti-ezrin polyclonal antibody (pAb) $(1: 1,000)$, and anti-actin mAb $(1: 1,000)$. Secondary Ab's coupled to peroxidase and chemiluminescence revelation (Invitrogen Sarl) were used.

Transmission electron microscopy. Morphological studies were performed as described previously (17). Briefly, epithelial spheroids were fixed with $2 \%$ glutaraldehyde during mucociliary differentiation in the absence or presence of a cytokine at different times during differentiation. They were postfixed in $1 \% \mathrm{OsO}_{4}$, dehydrated in ethanol, and embedded in Epon. Thin sections were examined on a JEOL electron microscope (JEOL SA, Croissy sur Seine, France).

cDNA array analysis. Total RNA was extracted from cells by the acid phenol/guanidine thiocyanate method of Chomczynski and Sacchi (18). PolyA ${ }^{+}$RNAs were isolated using an oligo-dT kit (QIAGEN SA Courtaboeuf, France) from epithelial cells at day 11 during MCD in the presence or absence of IL-13 from day 2. Differential hybridization analysis was achieved with CLONTECH Laboratories Inc. (Palo Alto, California, USA) Atlas cDNA arrays containing 588 selected cDNAs on a nylon membrane. Complex probes 
a

Human nasal cells dissociated from polyps or turbinates

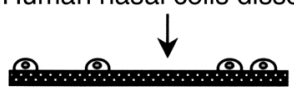

Proliferation

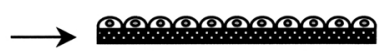

Confluence (C)

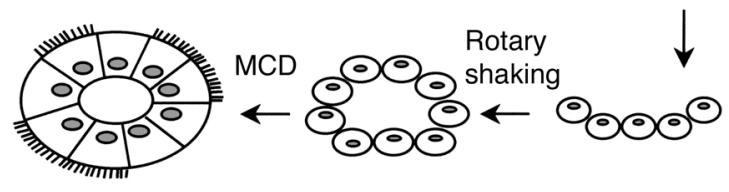

b
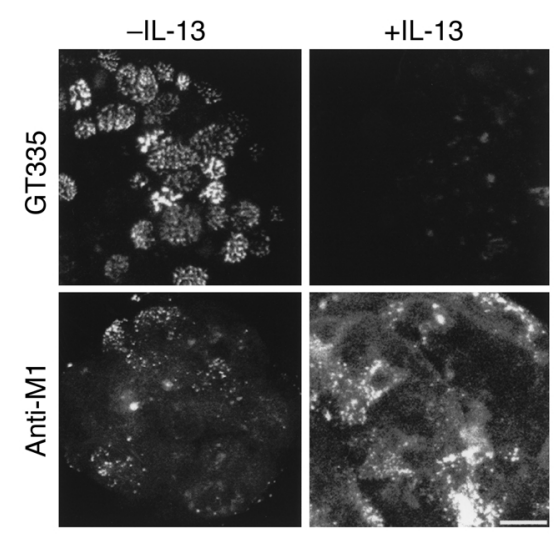

$c$

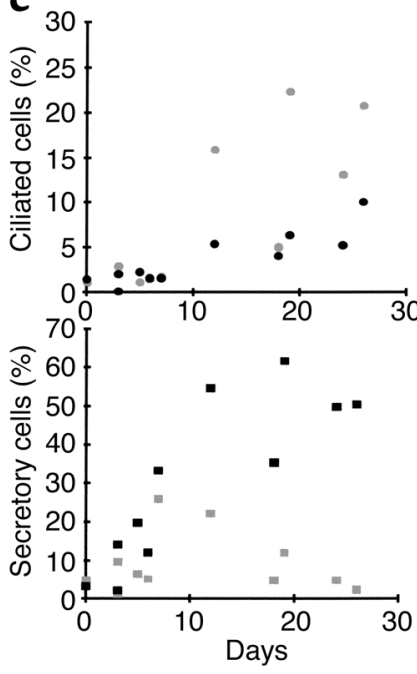

\section{Figure 1}

IL-13 modifies the proportion of differentiated phenotypes during MCD. (a) Scheme illustrating the different steps of the primary culture. Cells dissociated from human nasal polyps or turbinates are plated on thick collagen. Basal-like epithelial cells proliferate and form an epithelial monolayer. At confluence (C), collagenase removes epithelial cell sheets that are then rotary shaken to form epithelial spheroids. Epithelial cells then polarize and differentiate into secretory and ciliated cells. (b) Detection of ciliated cells (GT335) and secretory cells (Anti-M1) within epithelial spheroids during MCD in the absence (-IL-13) or in the presence (+IL-13) of the cytokine (by indirect IF). Note the absence of ciliated cells and the high number of secretory cells in the presence of the cytokine. Bar, $10 \mu \mathrm{m}$. (c) Quantification of IL-13 effect during MCD (flow cytometry). Cells dissociated from epithelial spheroids were analyzed using GT335 for ciliated cells and anti-M1 for secretory cells during the time course (0-30 days) of MCD. IL-13 $(10 \mathrm{ng} / \mathrm{ml})$ decreases the proportion of ciliated cells and largely increases the proportion of secretory cells. Three independent kinetics are shown. Gray symbols, -IL-13; black symbols, +IL-13. labeled with ${ }^{32} \mathrm{P}$ were obtained from subtractions (data not shown) or from total cDNAs and hybridized to the cDNA array (Atlas Human Cancer cDNA Expression Array; CLONTECH). Hybridization and washes were performed under highly stringent conditions.

Ciliary beat frequency analyses. Three-week-old suspension cultures of cells obtained from three different individuals (triplicate measurements per individual) were used with a continuous stimulation (1-6 days) with $0,1,10$, and $100 \mathrm{ng} / \mathrm{ml}$ of human recombinant IL-13. CBF was measured at room temperature $\left(22^{\circ} \mathrm{C}\right)$ using computerized microscope photometry as described previously (19). The CBF was measured on five different spheroids per flask. The measurements in triplicate gave us $75 \mathrm{CBF}$ values per individual.

\section{Results}

IL-13 stimulates goblet cell differentiation and inhibits ciliogenesis. In the absence of a human respiratory epithelial cell line able to differentiate in vitro, we developed primary cultures of HNE cells as initially described by Jorissen et al. (16). In that system, proliferation of basal-like epithelial cells precedes production of cell spheroids and further MCD (Figure 1a). To quantify ciliated and secretory cells, two specific mAb's were used: GT335 reacting with glutamylated tubulin (20), a component of cilia axonemes (14), and anti-M1, recognizing the product of the MUC5AC gene $(21,22)$, a differentiation marker of secretory cells $(23,24)$. These two Ab's allowed us to monitor the number of ciliated and secretory cells among the cell population (Figure 1b). After 1 week of MCD in standard culture conditions, the percentage of secretory cells reached $25 \%$, largely exceeding the number of ciliated cells. After 3 weeks of culture, the situation was reversed: about $25 \%$ of cells were ciliated whereas the number of mucin-positive cells was reduced approximately twofold (Figure 1c). Addition of IL-13 (10 ng/ml) to the medium induced both a drastic increase in the number of secretory cells and a reduction in the number of ciliated cells compared with control cultures (Figure 1, b and c).

IL-13 had a dose- and time-dependent effect (Figure $2 a)$. When added at the proliferation stage, it had little effect on the epithelial differentiation (Figure 2b). The presence of IL-13 was required during the whole MCD stage to develop a full-scale effect, because shorter treatments only resulted in partial inhibition of ciliated cell production (Figure 2c). Since the only known signaling pathway described for IL-13 includes a receptor shared with IL-4, we also evaluated the activity of IL-4 on human MCD. Figure 2d shows that IL-13 can be replaced by IL-4, but not by IFN- $\gamma$, to modify the course of epithelial cell differentiation. IFN- $\gamma$, in fact, had an inhibitory effect on IL-13-induced MCD (not shown). To further demonstrate that both IL-4 and IL-13 act through the same receptor, epithelial cells were simultaneously treated with IL-13 and increasing amounts of an IL-4 mutant (Y124D), a selective antagonist of IL-4/IL-13 shared receptor. Addition of the IL-4 mutant (Y124D) lead to a partial $(10 \mathrm{ng} / \mathrm{ml})$ or complete $(100$ $\mathrm{ng} / \mathrm{ml}$ ) reversion of IL-13 effects (Figure $2 \mathrm{e}$ ). Taken 
a

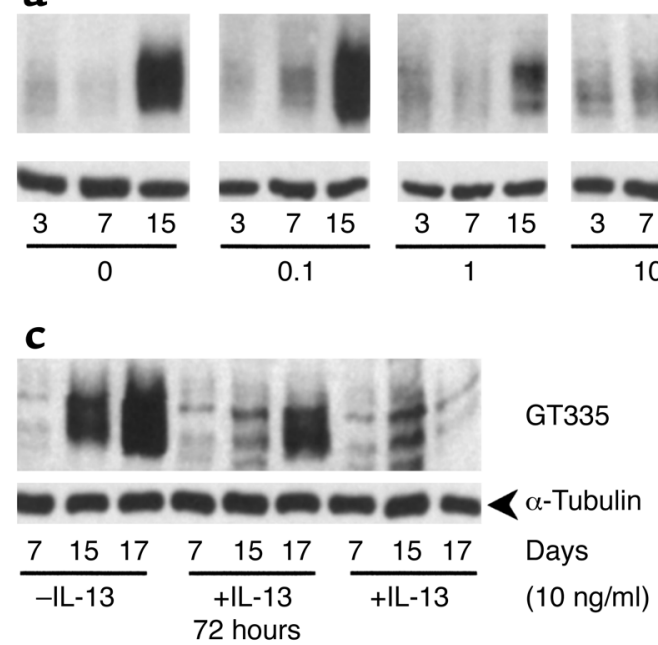

b

GT335

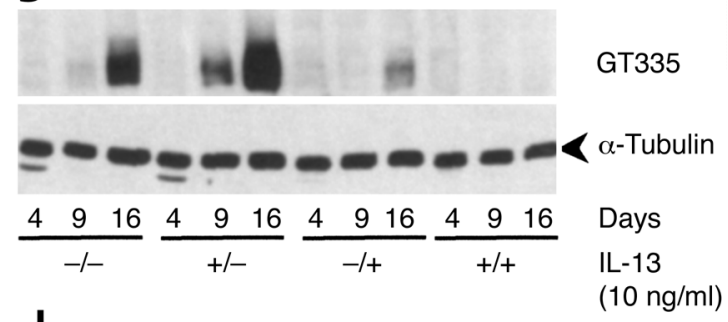

d

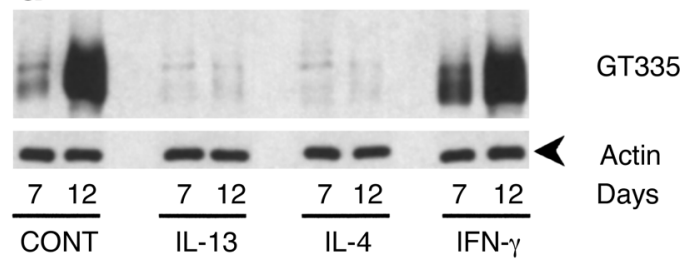

e

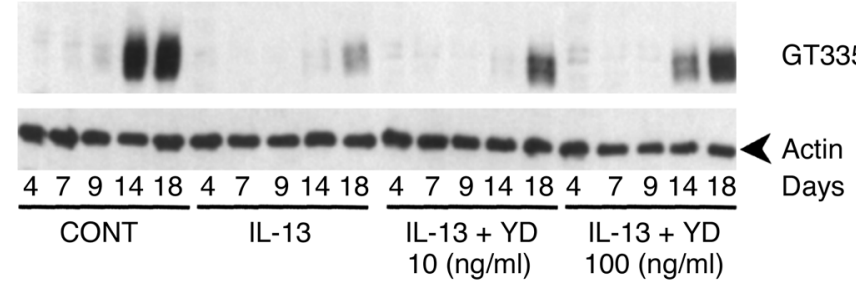

\section{Figure 2}

Dose- and time-dependent effect of IL-13 on MCD. GT335, a mAb that detects glutamylated tubulins, gives the proportion of ciliated cells during MCD in Western blot analysis (14). The indicated numbers represent the days after confluence. (a) Dose-dependent effect of IL-13 on ciliated cell differentiation. The proportion of ciliated cells at day 15 decreases with the dose of the continuously applied cytokine during differentiation. (b) Epithelial cells were continuously treated with IL-13 during proliferation (+/-), differentiation $(-/+)$, or both (+/+), compared with control cultures $(-/-)$. No effect is observed on ciliated cell differentiation when cells are treated with the cytokine during proliferation (+/-). The increased GT335 staining at day $9(-/+$ compared with $-/-)$ is not significant, varying from one experiment to another. Ciliated differentiation is strongly reduced when cells are treated during differentiation $(-/+$ or $+/+)$. (c) Epithelial cells were either treated for 72 hours (between day 4 and day 7$)$ or continuously treated with IL-13 $(10 \mathrm{ng} / \mathrm{ml})$. The maximum effect is observed for a continuous treatment. (d) Epithelial cells were continuously treated with IL-13, IL-4, or IFN- $\gamma(10 \mathrm{ng} / \mathrm{ml})$ during differentiation. IL-13 and IL-4 effects are identical, while IFN- $\gamma$ does not alter the ciliated cell differentiation process. (e) The IL-4 antagonistic mutant protein (Y124D) antagonizes the IL-13 effect in a dose-dependent manner. The same amount of total protein extracts were deposited per lane (10 $\mu \mathrm{g})$. Actin or tubulin were revealed as constant protein markers during the kinetics of differentiation.

together, these data demonstrate that, as in the mouse, IL-13 and IL-4 promote differentiation of human epithelial cells into goblet cells through a common signaling pathway, which includes IL-4R $\alpha$. Moreover, IL-13/IL-4 treatment of epithelial cells also alters ciliogenesis.

IL-13 impairs epithelial cell polarization. The polarized character of respiratory epithelial cells involves the actin cytoskeleton and membrane-associated protein complexes. In particular, molecular scaffolds from tight junctions are clearly implicated in the regulation of paracellular permeability. Since alterations of ciliogenesis suggested possible cytoskeletal modifications, we undertook a morphological study of epithelial cells during the differentiation process. IL-13-treated and -untreated spheroids were fixed at various stages of differentiation and analyzed using transmission electron microscopy. Several modifications in cell organization were observed at the ultrastructural level (Figure 3). IL-13 induced the formation of numerous interdigitations within lateral membranes (Figure 3, $\mathrm{d}$ and $\mathrm{h}$ ). Ramified microvilli were also frequently observed with- in apical membranes of IL-13-treated cells (Figure 3, d and f) compared with simple ones in untreated cells. In some cases, cilia of IL-13-treated cells were detected in nonapical membrane areas, suggesting a defect in basal body targeting (Figure $3 \mathrm{~g}$ ). In the control culture, basal bodies were anchored to the apical membrane among dispersed microvilli (Figure 3c). Furthermore, impairment of cell polarization was also suggested by the presence of abnormal microvilli within basal membranes of IL-13-treated cells (Figure 3, d and e).

IL-13 affects the expression and localization of ezrin. A differential cDNA analysis was performed to identify genes upregulated or downregulated during IL-13 treatment, using CLONTECH Laboratories Inc. The cDNA arrays, consisting of 588 selected cDNA on nylon membranes. Among the 588 clones, 137 clones were detected using probes from IL-13-treated cultures, untreated cultures, or both (data not shown). Interestingly, expression of several proteins involved in cell polarization was downregulated by IL-13: $\alpha$-catenin, plakoglobin, RhoA, G25K (a Cdc42 homologue belonging to the Rho small G pro- 

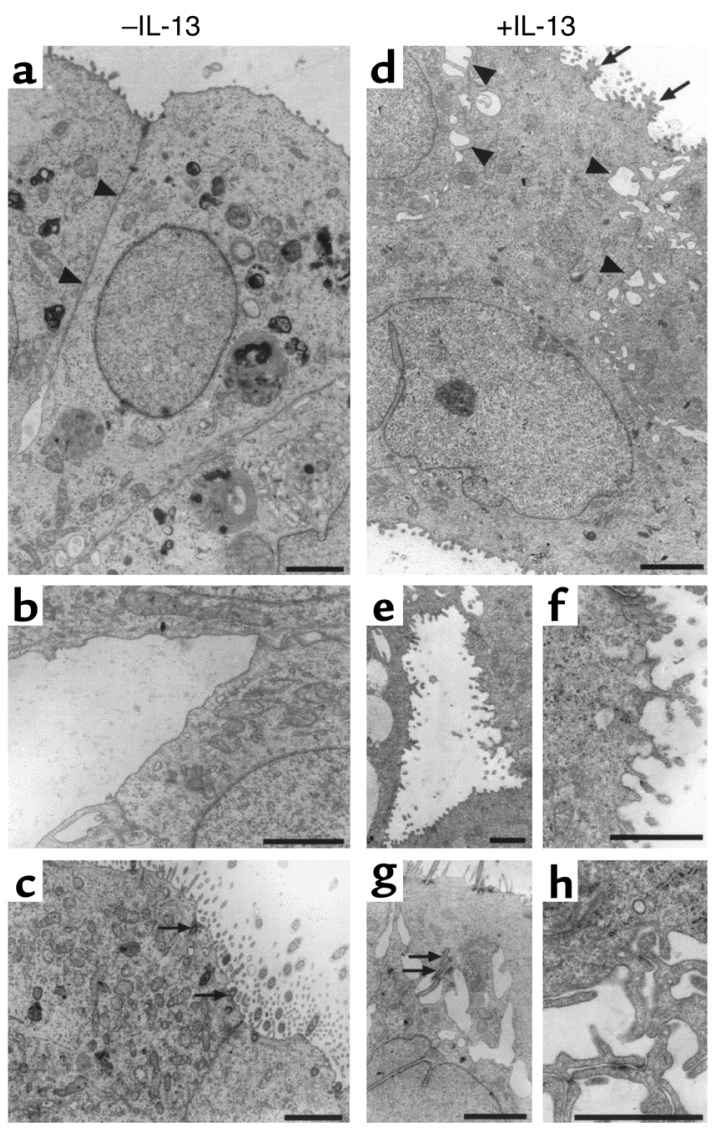

tein family) (data not shown), and ezrin (Figure 4a). Downregulation of ezrin expression by IL-13 was confirmed at the protein level (Figure $4 \mathrm{~b}$ ) and by RT-PCR experiments (data not shown). In addition, during the first days of in vitro differentiation, ezrin was localized

\section{Figure 4}

IL-13 affects ezrin expression and localization during mucociliary differentiation. (a) ${ }^{32} \mathrm{P}$-labeled complex probes were obtained from epithelial cell RNAs at day 11 during MCD (+/- IL-13 from day 2) and hybridized on CLONTECH Laboratories Inc.'s Atlas CDNA arrays. CD9, a member of the tetraspanin protein family, is strongly expressed in both conditions. Merlin is not detectable in either case, while ezrin expression is downregulated by IL-13. (b) Ezrin protein expression was downregulated by IL-13 $(+7,+12$ days after confluence during MCD). At day 7 , protein expression is identical in the two conditions. At day 12, the difference is significant. The Western blot analysis results shown are from one representative experiment, corresponding to a $30 \%$ decrease of ezrin expression in the presence of IL-13. Ten micrograms of total protein extract per lane. (c) Localization of ZO-1 and ezrin is affected by IL-13. Epithelial cells were labeled with GT335 mAb (red) in combination with either anti-ZO-1 Ab (green) or anti-ezrin Ab (green). In control conditions during MCD, anti-ZO-1 Ab reveals a continuous regular pattern, defining the subapical membrane domain (tight junctions) of each individual cell. IL-13-treatment strongly affects epithelial cell shape as observed with anti-ZO-1 staining. Anti-ezrin Ab decorates microvilli specifically located at the apical membrane of ciliated cells. In IL-13-treated spheroids, ezrin largely remains in the cytoplasm. Note the low number of ciliated cells (GT335 positive-cells) in the presence of the cytokine. Bar, $10 \mu \mathrm{m}$.

\section{Figure 3}

IL-13 alters the polarization of epithelial cells. Transmission electronic microscopy analyses were performed on epithelial spheroids during MCD in the absence $(\mathbf{a}, \mathbf{b}, \mathbf{c})$ or in the presence $(\mathbf{d}, \mathbf{e}, \mathbf{f}, \mathbf{g}, \mathbf{h})$ of IL-13. Epithelial cells from untreated spheroids display a close contact of lateral membranes (a) (arrowheads) compared with IL-13-treated cells (d). The latter exhibit alterations of the lateral membranes characterized by decreased cell-cell contacts, large intercellular spaces (d) (arrowheads), and interdigitations (h). These cells also possess an apical (d) or basal $(\mathbf{e}, \mathbf{f})$ membrane surface that is lined by a condensed microvilli compared with control cells presenting a more regular membrane surface. (a) Apical; (b) basal. In control conditions (day 15), centrioles were currently detected in epithelial cells, and mature basal bodies were anchored at the apical membrane among dispersed simple microvilli (c) (arrows). In some cases, a default in centriole/basal bodies targeting is observed in IL-13-treated spheroids (arrows in $\mathbf{g}$ ). Bars, $2 \mu \mathrm{m}$.

within the cytoplasm (data not shown), whereas it was specifically directed to the microvilli of ciliated cells (Figure 4 c) before the formation of cilia (data not shown). In IL-13-treated spheroids, ZO-1 detected an irregular pattern compared with control, and few cells displayed apical membrane ezrin localization compared with control conditions (Figure 4c).

Taken together, these data suggest that IL-13 impairs ciliogenesis, at least partly through modifications of expression and localization of components implicated in the process of epithelial cell polarity.

IL-13 decreases ciliary beat frequency. The morphological alterations observed in IL-13-treated epithelial cells suggested a possible inhibition of ciliary function, since this

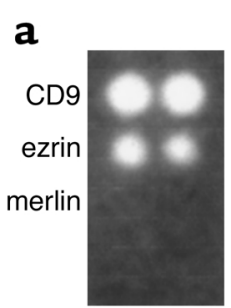

IL-13

C

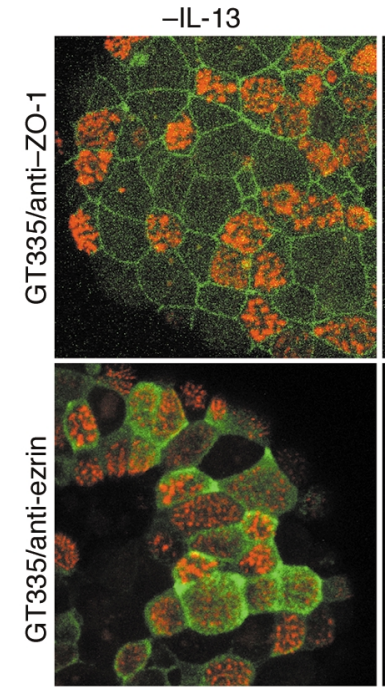

b

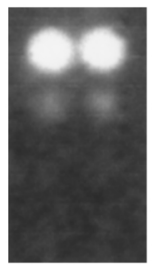

IL-13 -

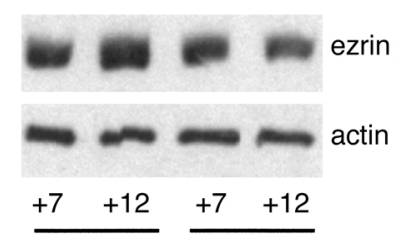

$+\mathrm{IL}-13$

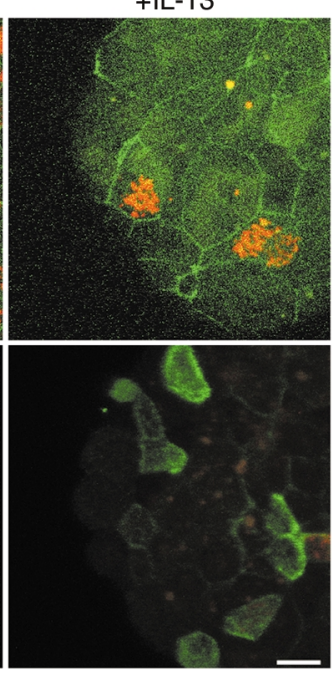



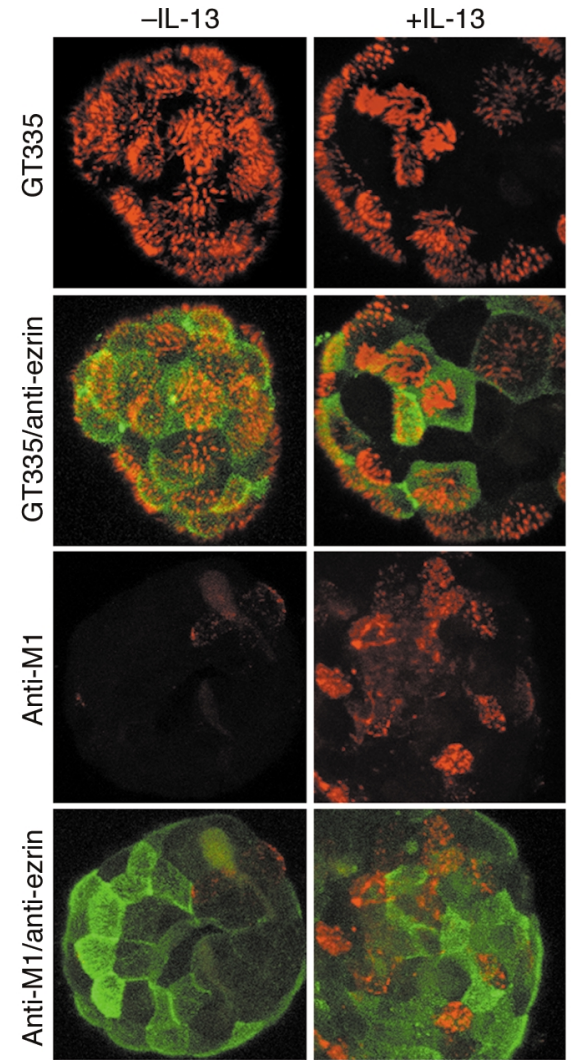

b

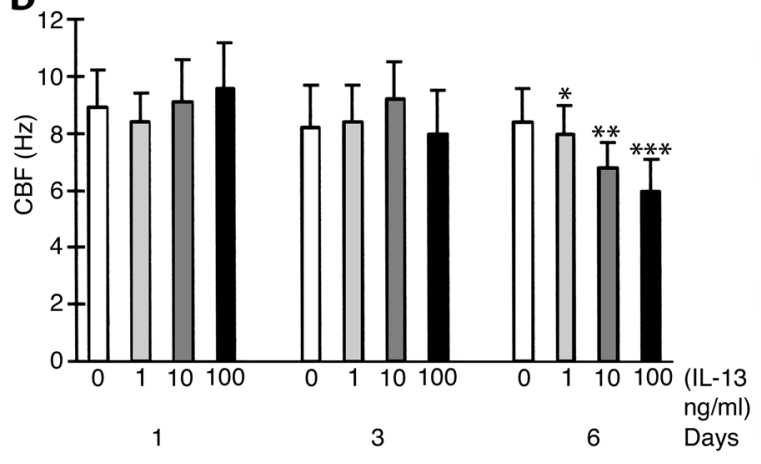

function relies on both a proper cellular cytoskeletal organization and cell-to-cell lateral adhesion. Therefore, ciliary beat frequency (CBF) was measured in IL-13-treated and -untreated conditions. In these experiments, the cytokine was added to spheroid cultures after 18 days of MCD, several days after the onset of ciliogenesis. During the first 3 days of IL- 13 treatment, no significant difference was observed in the number of ciliated cells between control and treated spheroids. After 6 and 11 days, the number of ciliated cells increased in the control, while it remained stationary in IL-13-treated spheroids (data not shown). In parallel, the cytokine induced marked increases of mucin-positive cells, as revealed by the anti-M1 staining (Figure 5a). Interestingly, a significant reduction of $\mathrm{CBF}$ was observed in a dose-dependent manner at day 6 of IL-13 exposure, but not at day 3 (Figure $5 \mathrm{~b}$ ). After 10 days of IL-13 treatment, ciliary beating was completely abolished (data not shown).

\section{Figure 5}

IL-13 effect on partially differentiated epithelial spheroids: IL-13 inhibits further ciliogenesis, increases the proportion of MUC5ACpositive cells, and decreases ciliary beat frequency. (a) Partially differentiated (ciliated) epithelial spheroids ( 18 days after confluence) were continuously treated with IL-13 for 11 days and processed for double IF analysis (day 29). The proportion of ciliated cells remained lower than in control conditions after IL-13 treatment (single staining, GT335: compare -IL-13 and +IL-13). Numerous MUC5AC-positive cells (Anti-M1) were observed in the IL-13-treated conditions compared with control conditions. Accordingly, the proportion of epithelial cells with ezrin located within the apical membrane domain was lower in the presence of the cytokine. Note that ezrin staining is absent in MUC5AC-positive cells (+IL-13, Anti-M1/anti-ezrin). Bar, $10 \mu \mathrm{m}$. (b) The effect of IL-13 on CBF was determined on partially differentiated spheroids (21 days in suspension after confluence). The CBF was measured after 1,3 , and 6 days. CBF does not change significantly after 1 and 3 days of continuous treatment with IL-13. A significant concentration-dependent downregulation of the $\mathrm{CBF}$ values is observed after 6 days of continuous stimulation $(0,1,10$, $100 \mathrm{ng} / \mathrm{ml}$ of IL-13). The values are expressed as mean \pm SD. The Student $t$ test was used to compare IL-13-stimulated cells and controls at the same incubation time. A value of $P<0.05$ was accepted as statistically significant $\left({ }^{*} P<0.02 ;{ }^{*} P<0.01 ;{ }^{*}{ }^{*} P<0.0001\right)$.

\section{Discussion}

IL-13 plays an important role in the pathogenesis of asthma and other Th2-polarized tissue responses. Alteration of the epithelial barrier induces an inflammatory process in which both Th1 and Th2 cytokines are clearly implicated. Because the inhibition of IL-13 activity is sufficient to reduce the asthma phenotype in mice, in particular goblet cell hyperplasia $(25,26)$, we tested the effect of the cytokine during MCD of HNE cells as a model of epithelial cell regeneration. We used a primary culture because no human respiratory epithelial cell line is able to differentiate into secretory and ciliated cells. No neutrophils and eosinophils are present in the culture, allowing us to detect direct effects of IL-13 on the differentiation of epithelial cells.

IL-13 increases the proportion of secretory cells. We have used the Muc5AC mucin gene product as a marker of secretory cell differentiation since an increase in its expression was correlated with an increase in mucus cells in murine airways, while the level of Muc1 mRNA and protein was constant (27). In addition, human tracheobronchial epithelial cells were shown to express increasing mRNA levels of the MUC5AC, MUC5B, and MUC4 genes during in vitro differentiation, while the levels of MUC2 mRNA remained low $(23,28,29)$. In this paper, we show that IL-13 largely increases the proportion of MUC5AC-positive cells during in vitro MCD. Interestingly, the same effect was observed when IL-13 was added on differentiated epithelial cells (see Figure 5). Thus, IL-13 is able to increase the percentage of mucus-positive cells in human cell cultures in vitro. In vivo, IL-13 upregulates MUC5AC, but not MUC2, expression (27). Previous studies using cultured epithelial cells have shown that IL-13 and IL-4 do not affect, or may even decrease, mucin gene expression (30-32) and that 
IL-4 upregulates MUC2 expression (33). Our cell culture conditions thus more closely reproduce the situation in vivo, in that MUC5AC expression is upregulated. Recent studies using a selective EGFR-tyrosine kinase inhibitor and cyclophosphamide or anti-IL-8 to block neutrophil recruitment (24) have suggested that EGFR signaling and neutrophils are involved in the IL- 13 effects on mucin production. Our cell cultures, in the absence of neutrophils, show that these cells are not essential for the IL-13 regulation of mucin production. Whether the effects of IL-13 are directly on mucus production or on the differentiation of mucus cells as suggested by Rose et al. (31) remains to be further investigated.

IL-4 also increased the proportion of MUC5AC-positive cells (data not shown). Both IL-13 and IL-4 have been shown to use the IL-4R $\alpha$ as a receptor component and thus activate common signaling pathways involving Janus kinases (JAKs), STAT6, and other signaling molecules (34) (see references therein). It is noteworthy that IL-4R $\alpha$ and STAT6 are critically important in Th2 cell stimulation of mucus production, because mucus was not produced in IL-4R $\alpha^{-/-}$mice inoculated with Th2 cells (35) and in STAT6 ${ }^{-/}$mice (11). The IL-13R in epithelial cells is composed of the IL- $4 \mathrm{R} \alpha$ subunit and an IL-13-binding subunit. Of the two IL-13-binding subunits, IL-13R $\alpha 1$ has been shown to be expressed in HNE cells (36) and to be implicated in IL-13 signaling (37). In HNE cells, the IL-4 antagonistic mutant protein (Y124D) antagonized IL-13 effects (see Figure 2), demonstrating the implication of a shared IL-4/IL-13 receptor in epithelial cells to IL-13. However, IL-4 appears to play a more important role in Th2 cell development and recruitment to the airways (2). Importantly, IFN- $\gamma$ was not found to interfere with the MCD process (see Figure 2), but instead, was able to reduce IL-13-induced goblet cell hyperplasia (data not shown). A similar result was reported recently in an in vivo mouse model (38).

IL-13 alters epithelial cell morphology and ciliated cell differentiation. The molecular basis involved in the process of ciliogenesis remains largely unknown. Tubulin glutamylation is a marker of cilia axonemes. GT335, a $\mathrm{mAb}$ directed against glutamylated tubulins, stains the centriole/basal bodies and the axonemes of ciliated cells (14). The use of this Ab allowed us to quantify by flow cytometry the percentage of ciliated cells during MCD (see Figure 1). The relative proportion of ciliated cells could also be determined using immunodetection of glutamylated tubulin from total protein extracts in Western blot analysis (ref. 14 and this work).

IL-13 clearly inhibited ciliated cell differentiation in vitro. At the ultrastructural level, it altered epithelial cell morphology by creating large intercellular spaces and lateral membrane interdigitations. Moreover, it affected the targeting of basal bodies and, consequently, a lower proportion of ciliated cells was observed as the cultures aged. A screening of mRNAs differentially expressed during MCD in the presence or absence of IL-13 showed that several components involved in cell polarization such as RhoA, G25K, $\alpha$-catenin, and plakoglobulin were down- regulated at the transcriptional level (data not shown). Ezrin was affected at the transcriptional level and at the protein expression level (see Figure 4). Moreover, IL-13 affected its apical membrane targeting during differentiation. Ezrin belongs to the ERM family and acts as a link between the plasma membrane and the actin cytoskeleton (39) and may serve, in respiratory epithelial cells, as a protein kinase $\mathrm{A}$ anchoring protein for protein kinase A-mediated phosphorylation of CFTR (40). Ezrin may also determine survival of epithelial cells (41). In addition, IL-13 has been shown to increase epithelial permeability by activating the phosphoinositide 3-kinase (PI 3-kinase) pathway (42) and to stimulate activation of the PI 3-kinase-dependent signaling cascade in HT-29 cells (43). Consequently, it will be of interest to test PI 3-kinase inhibitors for cell survival and differentiation of normal epithelial cells treated with IL-13. It is noteworthy that IL-13 both alters epithelial cell polarization and further ciliated cell differentiation and alters the beating of cilia.

Multiple IL-13 effects contribute to the asthma phenotype. There is growing evidence that IL-13 is strongly involved in the pathogenesis of asthma and causes AHR and eosinophilia to the adult murine lung $(25,26$, $44,45)$. In an inducible transgenic mouse overexpressing IL-13, the cytokine caused emphysema, mucus metaplasia, and inflammation (45). However, mucus cell overproduction may be not sufficient to explain airway obstruction. An important result coming from our in vitro studies is that IL-13 may both induce mucus metaplasia and alteration of ciliated cell differentiation and function. Ultrastructural studies and anti-ZO-1 staining of epithelial cells during MCD in the presence of IL-13 suggest a modification of paracellular permeability. Epithelial cells show several morphological alterations interfering with the process of mucociliary differentiation. Interestingly, when the cytokine was added on differentiated epithelial cells, both an increase in the proportion of secretory cells and a time- and dose-dependent decrease of CBF were observed. Further incubation (up to 10 days) with the cytokine completely abolished ciliary beating (data not shown). The reduction of CBF is statistically significant and differs from results using other cytokines, such as IL-1 $\beta$ and TNF- $\alpha$, which increased CBF after a 4- to 24hour treatment (46). Taken together, these results suggest that during in vivo epithelial repair, IL-13 may influence the recovery of a normal, mucociliary, phenotype. The CBF-inhibitory effect of IL-13 may be relevant in vivo because inhibition of ciliary beating may contribute to airway obstruction.

IL-13-induced mucus overproduction in respiratory airways has been described in vivo $(25,26)$, suggesting that it is crucial for allergen-induced airway hyperresponsiveness. Recent data demonstrate an important role for genetic variants of IL-13 in the development of asthma in humans, independent of IL-4 (47). Our data reinforce the idea that the role of the cytokine is important in human asthma. Thus, inhibiting the cytokine or its receptors in vivo may be relevant in chronic lung 
diseases such as asthma. The in vitro system we developed will be useful for testing biological or synthetic IL-13 antagonist components.

\section{Acknowledgments}

We thank Philippe Denoulet, Jacques Bara, and Monique Arpin for Ab's and helpful discussions. We also thank Thomas Küntziger, Matthieu Piel, and Gérard Géraud for helpful advice regarding confocal microscopy, Linda Martin for helpful discussions, and Jean-Claude Courvalin for the use of "Das Microscope." The members of Laboratoire de Cytophysiologie et Toxicologie Cellulaire and Sanofi-Synthélabo laboratories are warmly acknowledged for technical help and comments on the manuscript. This work was supported by a grant (GEMO/L/012/00/P) from Sanofi-Synthélabo to the Laboratoire de Cytophysiologie et Toxicologie Cellulaire.

1. Van der Velden, V.H.J., Savelkoul, H.F.J., and Versnel, M.A. 1998. Bronchial epithelium: morphology, function, and pathophysiology in asthma. Eur. Cytokine Netw. 9:585-597.

2. Corry, D.B. 1999. IL-13 in allergy: home at last. Curr. Opin. Immunol. 11:610-614.

3. Loots, G.G., et al. 2000. Identification of a coordinate regulator of interleukins 4,13 , and 5 by cross-species sequence comparisons. Science. 288: $136-140$.

4. Hancock, A., Armstrong, L., Gama, R., and Millar, A. 1998. Production of interleukin 13 by alveolar macrophages from normal and fibrotic lung. Am. J. Respir. Cell Mol. Biol. 18:60-65.

5. Minty, A., et al. 1993. Interleukin-13 is a new human lymphokine regulating inflammatory and immune responses. Nature. 362:248-250.

6. Zurawski, S.M., et al. 1995 . The primary binding subunit of the human interleukin-4 receptor is also a component of the interleukin-13 receptor. J. Biol. Chem. 270:13869-13878.

7. Keegan, A.D., et al. 1995. Similarities and differences in signal transduction by interleukin 4 and interleukin 13: analysis of Janus kinase activation. Proc. Natl. Acad. Sci. USA. 92:7681-7685

8. Murata, T., Husain, S.R., Mohri, H., and Puri, R.K. 1998. Two different IL-13 receptor chains are expressed in normal human skin fibroblasts, and IL-4 and IL-13 mediate signal transduction through a common pathway. Int. Immunol. 10:1103-1110.

9. Murata, T., Noguchi, P.D., and Puri, R.K. 1996. IL-13 induces phosphorylation and activation of JAK2 Janus kinase in human colon carcinoma cell lines: similarities between IL-4 and IL-13 signaling. J. Immunol. 156:2972-2978.

10. Gavett, S.H., et al. 1997. Interleukin-4 receptor blockade prevents airway responses induced by antigen challenge in mice. Am. J. Physiol. 272:L253-L261.

11. Kuperman, D., Schofield, B., Wills-Karp, M., and Grusby, M.J. 1998. Signal transducer and activator of transcription factor 6 (Stat6)-deficient mice are protected from antigen-induced airway hyperresponsiveness and mucus production. J. Exp. Med. 187:939-948.

12. Propst, S.M., Denson, R., Rothstein, E., Estell, K., and Schwiebert, L.M. 2000. Proinflammatory and Th2-derived cytokines modulate CD40mediated expression of inflammatory mediators in airway epithelia: implications for the role of epithelial CD40 in airway inflammation. J. Immunol. 165:2214-2221.

13. Matsukura, S., et al. 2001. Interleukin-13 upregulates eotaxin expression in airway epithelial cells by a STAT6-dependent mechanism. Am. J. Respir. Cell Mol. Biol. 24:755-761.

14. Million, K., et al. 1999. Polyglutamylation and polyglycylation of $\alpha$ - and $\beta$-tubulins during in vitro ciliated cell differentiation of human respiratory epithelial cells. J. Cell Sci. 112:4357-4366.

15. Laoukili, J., et al. 2000. Differential expression and cellular distribution of centrin isoforms during human ciliated cell differentiation in vitro. $J$. Cell Sci. 113:1355-1364.

16. Jorissen, M., Van der Schueren, B., Van den Berghe, H., and Cassiman, J.J. 1989. The preservation and regeneration of cilia on human nasal epithelial cells cultured in vitro. Arch. Otorbinolaryngol. 246:308-314.

17. Boisvieux-Ulrich, E., et al. 2000. Differential effects of several retinoid receptor-selective ligands on squamous differentiation and apoptosis in airway epithelial cells. Cell Tissue Res. 300:67-81.

18. Chomczynski, P., and Sacchi, N. 1987. Single-step method of RNA iso- lation by acid guanidinium thiocyanate-phenol-chloroform extraction. Anal. Biochem. 162:156-159.

19. Willems, T., and Jorissen, M. 2000. Correlations between ciliary structure and ciliary function. Acta Otorbinolaryngol. Belg. 54:299-308.

20. Wolff, A., et al. 1992. Distribution of glutamylated $\alpha$ - and $\beta$-tubulin in mouse tissue using a specific monoclonal antibody, GT335. Eur. J. Cell Biol. 59:425-432.

21. Bara, J., et al. 1998. Gastric M1 mucin, an early oncofetal marker of colon carcinogenesis, is encoded by the MUC5AC gene. Int. J. Cancer. 75:767-773.

22. Labat, C., et al. 1999. M1/MUC5AC mucin release by human airways in vitro. Eur. Respir. J. 14:390-395.

23. Bernacki, S.H., et al. 1999. Mucin gene expression during differentiation of human airway epithelia in vitro. Am. J. Respir. Cell Mol. Biol. 20:595-604.

24. Shim, J.J., et al. 2001. IL-13 induces mucin production by stimulating epidermal growth factor receptors and by activating neutrophils. Am.J. Physiol. 208:L134-L140.

25. Grünig, G., et al. 1998. Requirement for IL-13 independently of IL-4 in experimental asthma. Science. 282:2261-2263.

26. Wills-Karp, M., et al. 1998. Interleukin-13: central mediator of allergic asthma. Science. 282:2258-2261.

27. Zuhdi Alimam, M., et al. 2000. Muc-5/5ac mucin messenger RNA and protein expression is a marker of goblet cell metaplasia in murine airways. Am. J. Respir. Cell Mol. Biol. 22:253-260.

28. Voynow, J.A., et al. 1999. Neutrophil elastase increases MUC5AC mRNA and protein expression in respiratory epithelial cells. Am. J. Physiol. 276:L835-L843.

29. Thornton, D.J., et al. 2000. Characterization of mucins from cultured normal human tracheobronchial epithelial cells. Am. J. Physiol. 278:L1118-L1128.

30. Brown, C.D., Kilty, I., Yeadon, M., and Jenkinson, S. 2001. Regulation of 15 -lipoxygenase isozymes and mucin secretion by cytokines in cultured normal human bronchial epithelial cells. Inflamm. Res. 50:321-326.

31. Rose, M.C., et al. 2000. Model systems for investigating mucin gene expression in airway diseases. J. Aerosol. Med. 13:245-261.

32. Jayawickreme, S.P., Gray, T., Nettesheim, P., and Eling, T. 1999. Regulation of 15-lipoxygenase expression and mucus secretion by IL-4 in human bronchial epithelial cells. Am. J. Physiol. 276:L596-L603.

33. Dabbagh, K., et al. 1999. IL-4 induces mucin gene expression and goblet cell metaplasia in vitro and in vivo. J. Immunol. 162:6233-6237.

34. Jiang, H., Harris, M.B., and Rothman, P. 2000. IL-4/IL-13 signaling beyond JAK/STAT. J. Allergy Clin. Immunol. 105:1063-1070.

35. Mohrs, M., et al.. 1999. Differences between IL-4- and IL-4 receptor alpha-deficient mice in chronic leishmaniasis reveal a protective role for IL-13 receptor signaling. J. Immunol. 162:7302-7308.

36. Akaiwa, M., et al. 2001. Localization of human interleukin 13 receptor in non-haematopoietic cells. Cytokine. 21:75-84.

37. Miloux, B., et al. 1997. Cloning of the human IL-13R alpha1 chain and reconstitution with the IL4R alpha of a functional IL-4/IL-13 receptor complex. FEBS Lett. 20:163-166.

38. Ford, J.G., et al. 2001. IL-13 and IFN-gamma: interactions in lung inflammation. J. Immunol. 167:1769-1777.

39. Gautreau, A., Louvard, D., and Arpin, M. 2000. Morphogenic effects of ezrin require a phosphorylation-induced transition from oligomers to monomers at the plasma membrane. J. Cell Biol. 10:193-203.

40. Sun, F., Hug, M.J., Bradbury, N.A., and Frizzell, R.A. 2000. Protein kinase A associates with cystic fibrosis transmembrane conductance regulator via an interaction with ezrin. J. Biol. Chem. 12:14360-14366.

41. Gautreau, A., Poullet, P., Louvard, D., and Arpin, M. 1999. Ezrin, a plasma membrane-microfilament linker, signals cell survival through the phosphatidylinositol 3-kinase/Akt pathway. Proc. Natl. Acad. Sci. USA. 22:7300-7305.

42. Ceponis, P.J., Botelho, F., Richards, C.D., and McKay, DM. 2000. Interleukins 4 and 13 increase intestinal epithelial permeability by a phosphatidyl 3 kinase pathway. J. Biol. Chem. 275:29132-29137.

43. Wright, K., Kolios, G., Westwick, J., and Ward, S.G. 1999. Cytokineinduced apoptosis in epithelial HT-29 cells is independent of nitric oxide formation. Evidence for an interleukin-13-driven phosphatidylinositol 3-kinase-dependent survival mechanism. J. Biol. Chem. 274:17193-17201.

44. Zhu, Z., et al. 1999. Pulmonary expression of interleukin-13 causes inflammation, mucus hypersecretion, subepithelial fibrosis, physiologic abnormalities, and eotaxin production. J. Clin. Invest. 103:779-788.

45. Zheng, T., et al. 2000. Inducible targeting of IL-13 to the adult lung causes matrix metalloproteinase- and cathepsin-dependent emphysema. $J$. Clin. Invest. 106:1081-1093.

46. Rhee, C.S., et al. 1999. Effects of IL-1 beta, TNF-alpha, and TGF-beta on ciliary beat frequency of human nasal ciliated epithelial cells in vitro. $A m$ J. Rhinol. 13:27-30.

47. Heinzman, A., et al. 2000. Genetic variants of IL-13 signalling and human asthma and atopy. Hum. Mol. Genet. 9:549-559. 